\title{
Dependence of mesoporous silica properties on its template
}

\begin{abstract}
Mesoporous silica has garnered a lot of attention since its discovery and this is due to their interesting properties such as high surface area, tunable pore size and facile functionalization. All these properties can be varied through its templates and synthesis parameters. Two of the most commonly used templates to synthesize mesoporous silica are cetyltrimethylammonium bromide (CTAB) and polymer PF-127. However, it was also found that ionic liquids (ILs) are also able to serve as template in mesoporous silica production. ILs are defined as salt that has melting point below $100{ }^{\circ} \mathrm{C}$ and one of the most interesting properties of ILs is the alteration of its anions will change its properties. Therefore, it is predicted that synthesizing mesoporous silica using ILs with various anions will alter the properties of mesoporous silica develop. Hence, in this research, we utilize long chain pyridinium ILs with various anions to synthesize mesoporous silica. It was found that the templates with different anions exhibited various morphologies such as spherical, distorted spherical, a type of raspberry and undefined shape with surface area in between $92.65 \mathrm{~m} 2 \mathrm{~g}-1$ to $494.95 \mathrm{~m} 2 \mathrm{~g}-1$ and the pore volume up to $1.333 \mathrm{~cm} 3 \mathrm{~g}-1$.
\end{abstract}

Keyword: Pyridinium; Ionic liquids; Anions; Mesoporous silica 